\title{
Electro-Search Algorithm for control in Position Tracking of DC Servo Motor
}

\author{
Tarek Hassan Mohamed \\ Electrical Engineering Department, \\ Faculty of Energy Engineering, Aswan University \\ Egypt \\ tarekhie@aswu.edu.eg
}

\author{
Atef Abdelfattah Atef \\ Electrical Engineering Department, \\ Faculty of Energy Engineering, Aswan University \\ atefabdelfatah2929@yahoo.com
}

\begin{abstract}
Industrial processes from longer time utilized servo motors .consequently Controlling the position of Direct Current servo motor is the first aim of this paper, During operation, happen disturbance in accuracy of position and velocity servo plant .consequently the best proportional velocity (PV) controller and natural phenomenal, named Electro-search(ES) algorithm for servo plant is used in improve position tracking of servo plant .proportional -velocity $(\mathrm{PV})$ is closed loop system have two terms: one to the position error (Kp) and the other used to the derivative of actual position $(\mathrm{Kv})$ of the plant . Electro-search (ES) algorithm based on the movement of electrons through the orbits around the nucleus of an atom .where natural of the electrons goes to orbits with high-rise energy level (better fitness value). This method depends on atomic structure and used physical laws such as Rydberg formula and Bohr model. The efficiency of( ES) algorithm compared with other famous optimization methods such as water cycle algorithm(WCA).this tested achieve by using MATLAB/SIMULINK. Result of simulation offer that (ES) algorithm high speed, high accuracy and efficient in improving characteristic of step response.
\end{abstract}

Keywords- DC servo motor; proportional-velocity (PV) controller; Electro-search algorithm.

\section{INTRODUCTION.}

No one will deny that DC machines little low size, physical strength, as a result, they're used in several industrial and business applications, including automatic production, exactness turntable devices, electrical wheel chairs, elevators, electrical bicycles[1]. this motors is wide used as a result of it comes in several shapes and sizes, in order that its application is sort of straightforward and versatile, and high torque and low price. Speed and position control square measure needed in industrial applications, robot manipulators and residential appliances [2]. Recently, servo motors are wide used as robotics manipulation, mechanism for automation management method, mechanical motion and direct drive application. Thus, we have a tendency to consideration servo motor for position tracking as a result of DC motor has some limitation to exactness activity of position instead of speed. For servo motor, rotor inertia area unit terribly tiny and time constant area unit extraordinarily tiny.
Thus the results of motors has terribly high torque-to-inertia ratios for commercially on the market application. Thus, gift work is predicated on DC servo motor SISO system for position management mistreatment management tool MATLAB.[3-5]Then efficiency of the planning is checked by MATLAB/Simulink. There are many methods to control position and velocity of DC servo motor such as sliding mode controller(SMC),2DOF PID, PID, water cycle algorithm(WCA), MPC, fuzzy logic, Electro-search algorithm(ES),Particle Swarm Algorithm (PSO), Grey Wolf Optimizer Algorithm ...etc.

[6-13]. proportional -velocity (PV) controller utilized in industrial process systems. Because it is need small knowledge, simple structure, information and little cost. Electro-Search (ES) algorithmic program incorporates some physical principals like Bohr model and Rydberg constant formula, adopting a three-phase theme. Within the atom spreading part, the atoms (i.e., candidate solutions) square measure every which way unfolds everywhere the molecular area (search space). In the orbital transmission part, the electrons rise to big orbits, aiming for orbits with towering energy levels (higher fitness value). The atoms square measure then resettled towards the world optimum purpose within the atom resettlement part, navigated by different atoms' flight. Besides, the (ES) calibration parameters are updated through sequent iterations via a self-tuning approach developed, particularly Orbital-Tuner method (OTM). The objective of this research of the servo plant is to obtain robustness versus system parameter differences, outer disturbances and realize desired position tracking [14-16].

This paper is organized as follows. Section II, III. Describes the mathematical model of DC servo motor and position velocity control IV. Presents the design of the optimization method electro-search control. Results and discussion are presented in V. Finally, conclusions are written in VI.

\section{SYSTEM DYNAMIC MODEL}

This servo plant are linear motion contain a cart paying by a DC motor, via the rack and pinion mechanism to ensure continuous traction. The car is also equipped with a rotary joint to which a free turning pendulum can be attached .both cart position and pendulum angle are sensed by encoderat fig. 1 


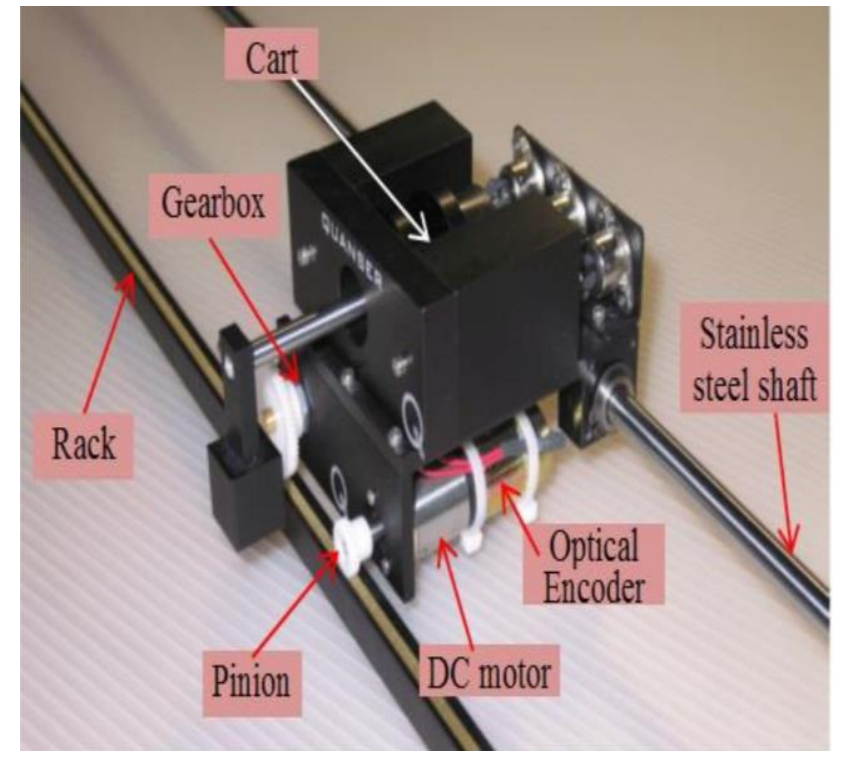

Fig. 1 Servo System Description

To derive G(s) used newton's second law of linear motion: $M\left(\frac{d^{2}}{A^{2}} x(t)\right)=F_{z}(t)-B_{x a}\left(\frac{d}{d x} x(t)\right)$

Just now, inertial force due to motor's armature in rotation ignored as well as the cart's coulomb friction is also ignored. Driving force Fc, produced by the DC motor and acting on the cart through the motor pinion expressed as:

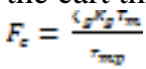

From electric circuit diagram of DC motor figure (2). Using Kirchhoff's voltage law:

$V_{\mathrm{m}}-R_{m} I_{m}-L_{m}\left(\frac{E_{i}}{I_{m}} I_{m}\right)-E_{\mathrm{sm} f}=0$

Since $<<R m$, the following equation:

$I_{m}=\frac{r_{m}-r_{k m v}}{R_{m}}$

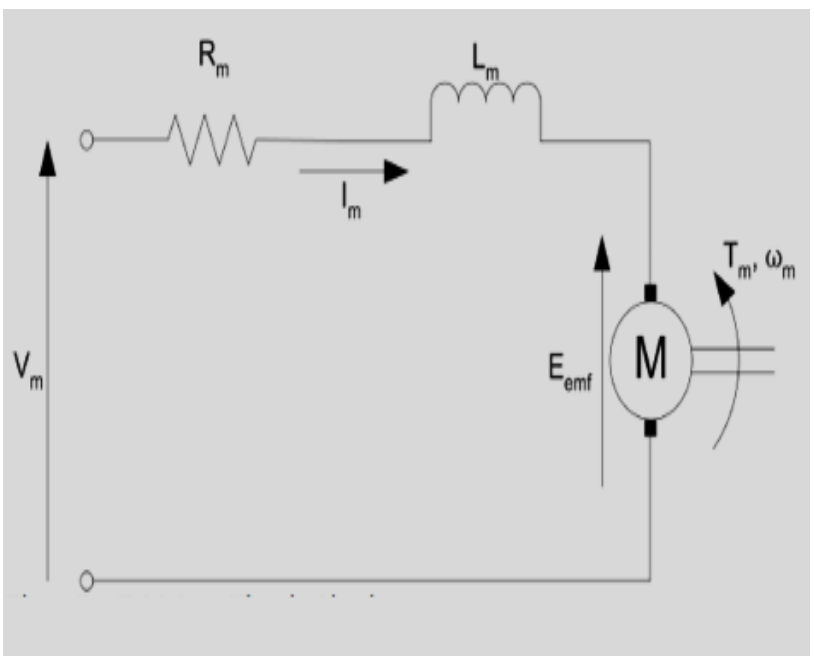

Fig .2 the armature circuit of the DC motor

The back-EMF voltage formed by the motor $(E e m f)$ is proportional to motor shaft velocity $(\omega m)$ :

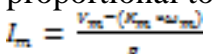

Furthermore, in order that account the DC servo motor electrical losses, the efficiency of motor is inserting to calculate the torque generated by the DC servo motor:

$T_{m}=\zeta_{m} K_{t} I_{m}$

(6)
Put Equations (5) and (6) into Equation (2) leads to:

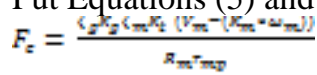

The motor angular velocity can be written such as

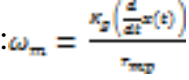

Finally the transfer function of servo system such that:

$G(s)=$

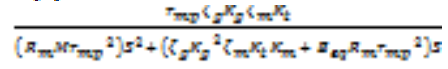

By using the model parameters values:

$G(s)={ }^{2.46} /(s+17.13) s$

\section{POSITION VELOCITY CONTROL}

PV control is presented at Figure (3). This control introduce two corrective terms: one is proportional by $(\mathrm{Kp})$ to position error and the other is proportional by $(\mathrm{Kv})$ this is velocity derivative of the actual position of DC servo plant. Equation (10), below, express the PV control law where Xd is the reference signal which is the desired position to track [17].

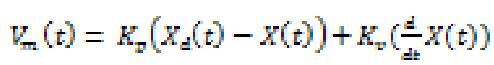

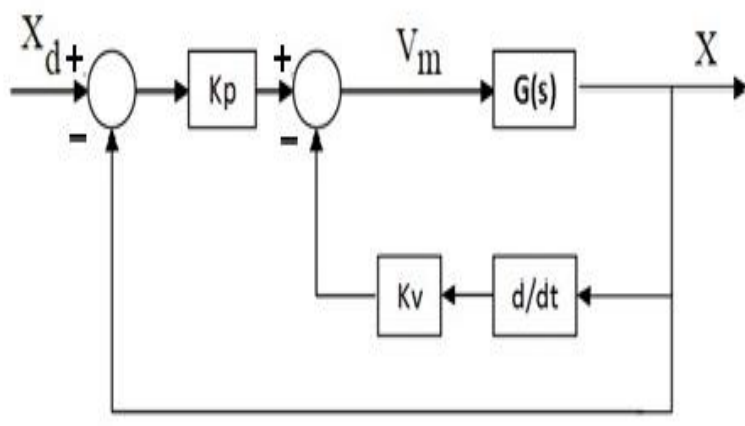

Figure .3 Simple constructions for PV controller

\section{ECTRO-SEARCH ALGORITHM}

In the Electro-Search algorithmic program, the domain of candidate solutions is analogous to the molecular area wherever numerous atoms positioned. The electrons round nucleus of every atom vary their orbits step by step so as to realize molecular states characterized by the very best energy level, that is analogous to the optimum point of the target operate. 
The Electro-search (ES) algorithm has three phases:

\section{A. 1 st phase atom spreading}

At this section, the candidate solutions are every which way distributed over the search house. Every candidate represents AN atom, composed of a nucleus around that the electrons orbit. The electrons are confined into outlined orbits the round of nucleus and will transition between them by absorbing or diffuse specific amounts of energy within the method (Bohr Model).[16]

\section{B. 2nd orbital transition}

In this section, the electrons around every nucleus move to larger orbits, aiming for orbits with higher energy levels. This orbital transition is performed by the construct of the quantity energy (Bohr, 1913). [16] The mechanics of quantum is energy of the particles within the model Bohr atom is restricted to a separate set of values or "quantized" levels. At this figure (5). Display quantity energy levels of hydrogen atom.

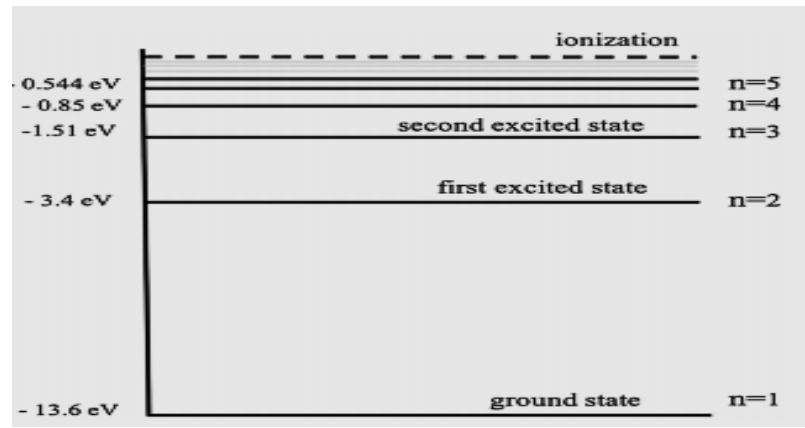

Fig .5 Quantized energy levels in hydrogen

This figure (6) .display atomic excitation happened through absorption of a photon as well as de-excitation happened by emission of photon (Lerner and Trigg, 2005; Parker, 1993) [17-18].

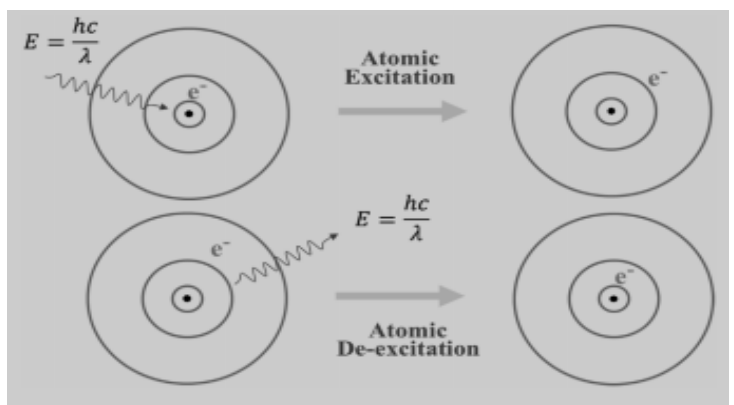

Fig.6.Electron excitation and de-excitation

Levels at hydrogen atom (see fig.2) expressed for each nucleus as follows:

$\theta_{i}=N i+(2 \times$ rand -1$)\left(1-\frac{1}{m^{2}}\right)$
$n \in\{2,3,4,5\}$

rand $E[0,1]$

where Ni mean that current position of nucleus rand used as uniform random numbers at rang $[0,1]$

$\mathrm{n}$ is the energy level

\section{3rd phase nucleus relocation}

In this step at figure (5) the position of the new nucleus $\left(N_{\mathrm{new}}\right)$ is assigned based on the energy of an emitted photon, defined by the difference of energy level between the two atoms (Rydberg formula). The equation form of nucleus relocation:

$D_{k x}=\left(e_{b x y t}-N_{b r a t}\right)+R_{x_{k}} \times\left(\frac{1}{g_{k x i}^{2}}-\frac{1}{w_{k}^{2}}\right)(3)$

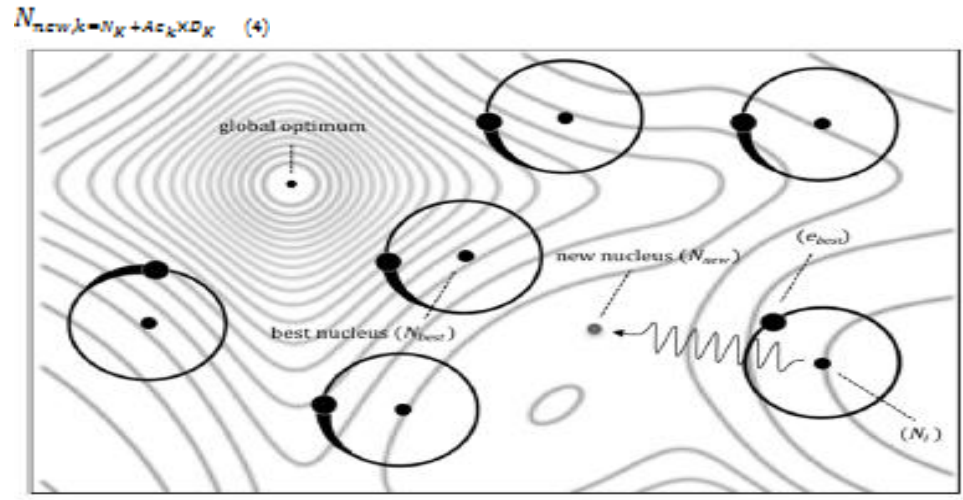

Fig. 4 the $3^{\text {rd }}$ phase of Electro-search (nucleus relocation)

During various iteration $\mathrm{k}$, relocation of distance $\left(D_{k}\right)$ are count for each nucleus based on the current better nucleus position $\left(N_{b z a x}\right)$ the better electron round nucleus ( $\left.\varepsilon_{\mathrm{brax}}\right)$ and the currently position of the nucleus $\left(N_{k}\right)$ influenced by constant $\left(\mathrm{R} \varepsilon_{k}\right)$ of Rydberg's energy. The position of the modern nucleus $\left(N_{\text {new }}\right)$ are updated utilize current position, and the count relocation distance. Influenced by coefficient of accelerator $\left(A c_{k=}\right)$.

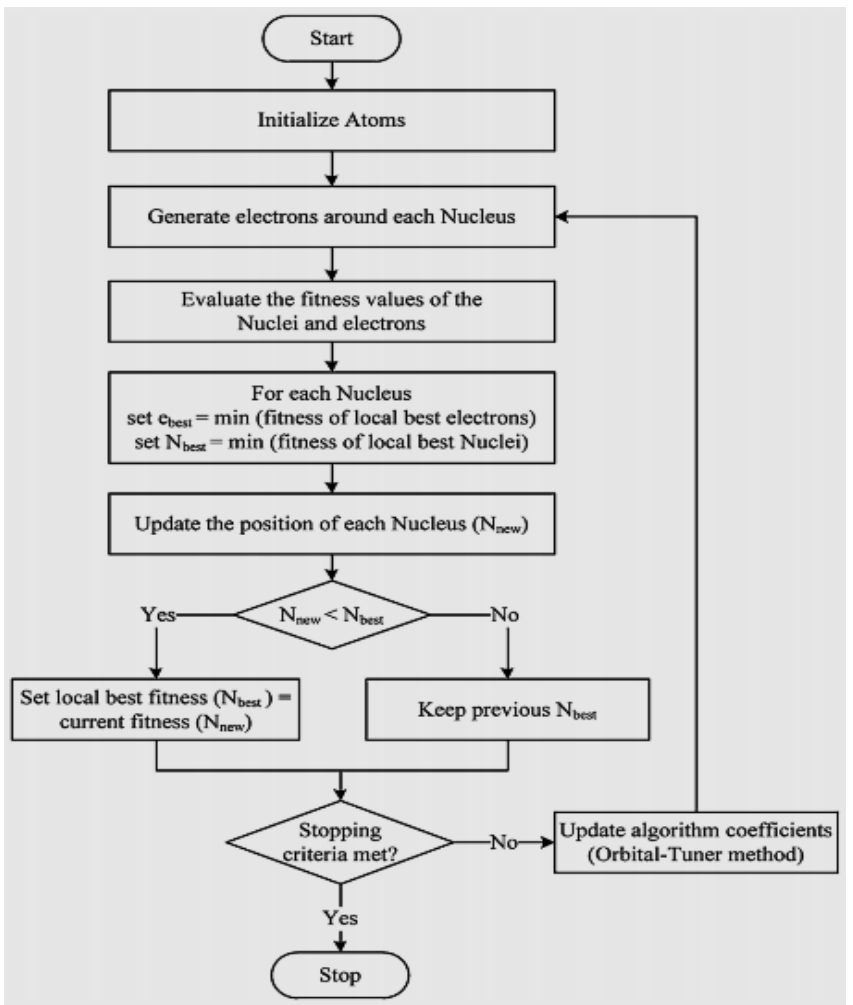

Fig .7 flow chart of the Electro-search 


\section{RESULTS AND DISCUSSIONS}

at this half, the structure of servo plant is planning at MATLAB appear at figure.(8), (PV) controller exploitation to enhance position tracking of servo plant .when this controller applying to the current plant to up characteristic of step response. This controller given terribly giant settling time, high rise time, higher peak overshoot this shown in figure (9)

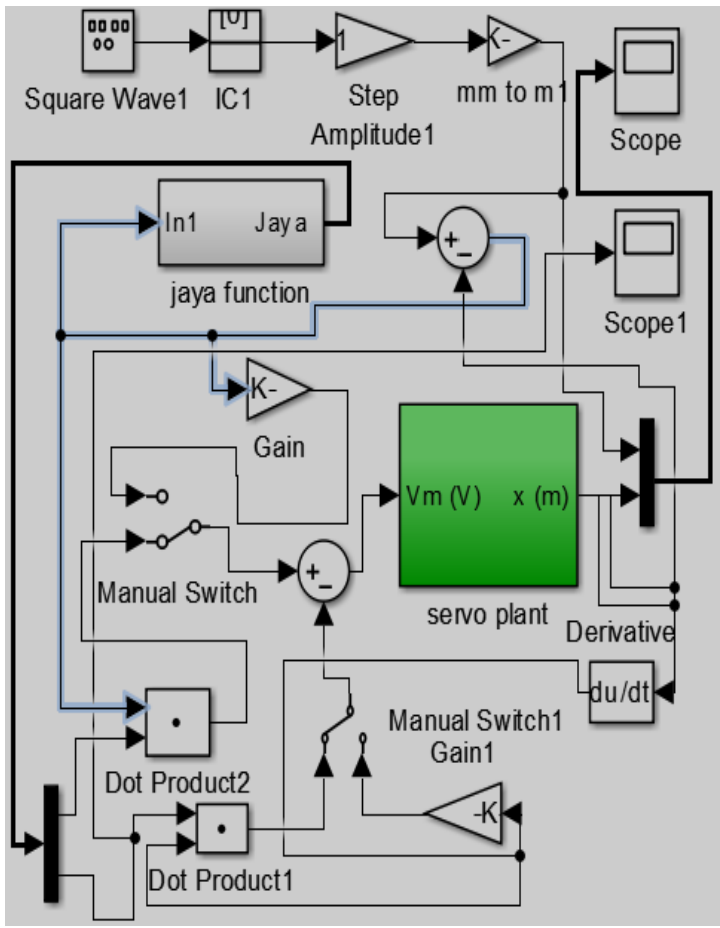

Fig.8 Simulink model for DC servo system

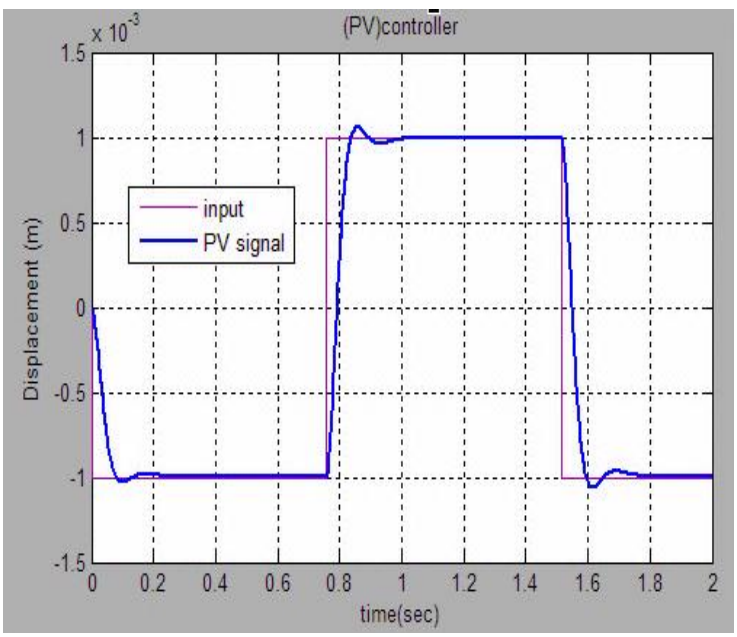

Fig. 9 simulation results of (PV) controller

Furthermore, PV controller has smart performance however not best for top performance system. So that , we tend to applied alternative management strategy named Electrosearch algorithmic program (ES) for DC servo plant to boost position tracking of DC servo motor figure.(10).

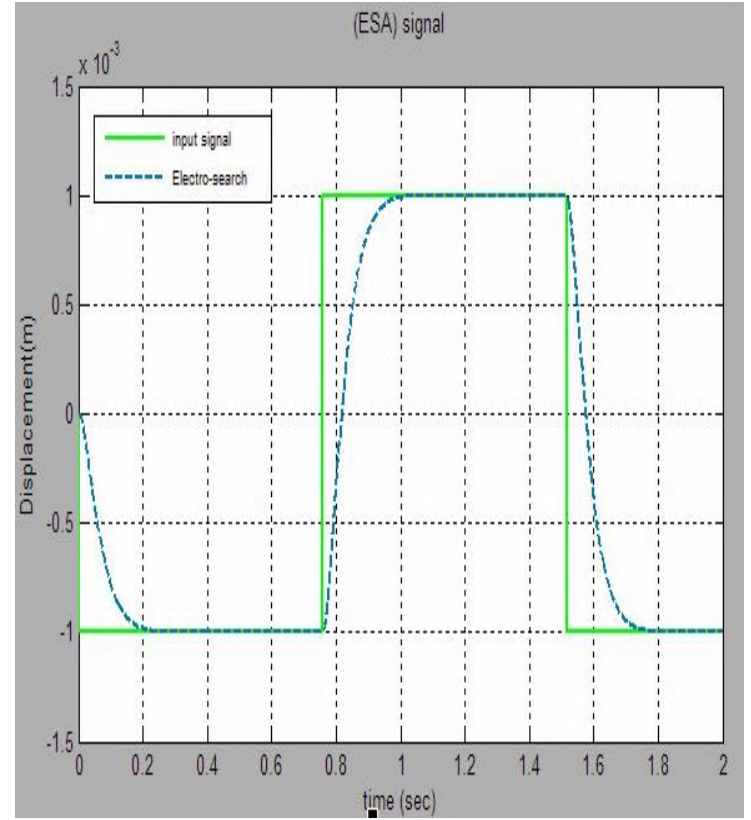

Fig.10 simulation results of Electro-search (ES)

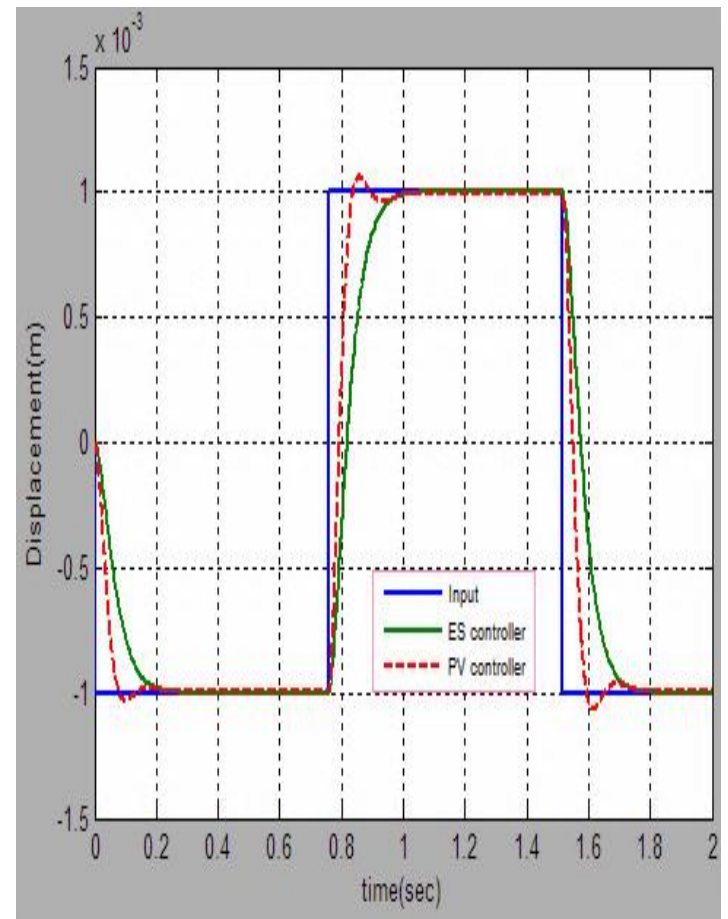

Fig.11 results of (PV) and (ES) controller

Simulation results in figure(11), show that Electro-search controller achieving optimal design for servo plant than (PV) controller because Electro- search algorithm has the best robustness and made optimization for characteristic of step response like very little settling time as well as peak overshoot is $0(\%)$. So (ES) controller gives higher effectiveness and accuracy when it compare with the (PV) controller.

\section{VI.CONCLUSIONS}

This paper present control strategy which uses (PV) controller and Electro- search algorithm (ES) are done in MATLAB /SIMULINK for achieving optimal design in the plant. the use of (PV) algorithm for control does not 
International Journal of Applied Energy Systems, Vol. 2, No. 2, July 2020 ISSN: 2636 - 3712 (Printed Version) ISSN: 2636 - 3720 (Online Version) Special Issue: ICEE-2019

achieving optimal control of system stability .furthermore, we use the higher performance control named Electrosearch algorithm to achieve the best system performance stability. Electro-search(ES) algorithm achieving optimal design because it has efficiency in improving characteristic response such as very less settling time, peak overshoot is $0(\%)$ and very small rising time.

\section{REFERENCES}

[1] D. B. Fogel, Evolutionary Computation: Toward a NewPhilosophy of Machine Intelligence. IEEE Press, Piscataway, New York, (1999)

[2] N.I. Ali, "Simulation for Position Control of DC Motor using Fuzzy Logic Controller," MSC thesis, Faculty of Electrical and Electronics Engineering, University Tun Hussein On Malaysia, 2013.

[3]Sidney R. Bowes, Fellow, Derrick Holliday "New Natural Observer Applied to Speed-Sensor less DC Servo and Induction Motors" IEEE Transaction Industrial Electronics, Vol. 51, No. 5, pp. 1025-1032, October - 2004. [4]Gwo-Ruey Yu, Ming-Hung Tseng and Yuan-Kai Lin "Optimal Positioning Control of a DC Servo Motor Using Sliding Mode" IEEE Conference Control Applications, pp, 272-277 September - 2004 [5]Ali Mohamed Yousef "Experimental Set up Verification of Servo DC Motor Position Control Based on Integral Sliding Mode Approach" Wseas Transaction on Systems and Control, Vol. 7, pp. 87-96, July - 2012, Egypt

[6] V.M. Alfaro, R. Vilanova, Model reference based robust tuning offive-parameter 2DOF PID controllers for first-order plus dead-time models, in: Proc. European Control Conference (ECC2013), Zurich, Switzerland, 2013,pp. 3931-3936.

[7] Araki, Mituhiko., and Taguchi, Hidefumi. (2003).TwoDegree-of-Freedom PID Controllers, International Journal of Control, Automation, and Systems, Vol. 1, No.4, 401-411.

[8] AshwaqAbdulameer, MarizanSulaiman, Mohd Aras, MohdShahrieel\& Dawood Saleem. " Tuning Methods of PID Controller for DC Motor Speed Control. " Indonesian Journal of Electrical Engineering and Computer Science. 3 (2016) 343349.

[9]Khaled sailan " DC Motor Angular Position Control using PID Controller for the porpuse of controlling the Hydraulic Pump " International Conference on Control, Engineering \& Information Technology (CEIT'13) Proceedings Engineering \& Technology - Vol.1,pp. 22, 26, 2013.

[10]A. Jalilvand, A. Kimiyaghalam, A. Ashouri, H. Kord " OPTIMAL TUNING OF CONTROLLER PARAMETERS ON A DC MOTOR BASED ON ADVANCED PARTICLE SWARM OPTIMIZATION ALGORITHM " "Technical and Physical Problems of Engineering" (IJTPE) December 2011 Issue 9 Volume 3 Number 4 Pages 10-17

[11] Kawai, Fukiko., et al,(2007). Automatic Tuning for Model Predictive Control: Can Particle
Swarm Optimization find a better parameter?, IEEE International Symposium on Intelligent Control - ISIC , pp. 646-651.

[12] Abhinav, R., Sheel, S.: "An adaptive, robust control of DC motor using fuzzy-PID

controller", Power Electronics, IEEE International Conference on Drives and Energy Systems, Dec. 2012.

[13]AliMadadi and Mahmood MohseniMotlagh " Optimal Control of DC motor using Grey Wolf Optimizer Algorithm "Technical Journal of Engineering and Applied Sciences C2014TJEAS Journal-2014-4-04/373-379ISSN 2051-0853

[14] Biegler, L.T., Grossmann, I.E., 2004. Retrospective on optimization. Computers and Chemical Engineering 28, 1169-1192.

[15] Boggs, P.T., Tolle, J.W., 2000. Sequential quadratic programming for large-scale nonlinear optimization. Journal of Computational and Applied Mathematics 124, 123-137.

[16]Bohr, N., 1913. On the constitution of atoms and molecules. Part I. Philosophical Magazine 26, 1-24.

[16] Cha, J.Z., Mayne, R.W., 1989. Optimization with discrete variables via recursive quadratic programming: part 1 - concepts and definitions. Transactions of the American Society of Mechanical Engineers 111, 124-129.

[17]Lerner, R.G., Trigg, G.T., 2005. Encyclopedia of Physics, 3rd ed. Wiley-VCH, Weinheim.

[18] Parker, S.P., 1993. McGraw-Hill Encyclopedia of Physics, 2nd ed. McGraw-Hill, New York.

[19] Tarek Hassan Mohamed, Essam Hafez Abdelhamid, Ammar Mostafa Hassan " Real Time Robust Position Controller for a Cart Moved by a DC Motor through "16th International Middle- East Power Systems Conference MEPCON'2014 Ain Shams University, Cairo, Egypt, December 23 - 25, 2014 [1] 